\title{
Research on GPS Receiver Data Processing Algorithm Based on Wavelet Analysis
}

\author{
Ershen Wang ${ }^{1,2}$, Tao Pang ${ }^{1}$ and Yongming Yang ${ }^{1}$ \\ ${ }^{1}$ Shenyang Aerospace University, School of Electronic and Information \\ Engineering, Shenyang, China \\ ${ }^{2}$ Beihang University, School of Electronic and Information Engineering, Beijing, \\ China \\ wes2016@126.com,pangtao163@126.com,995427179@qq.com
}

\begin{abstract}
Reliability is an essential performance in GPS navigation system. Therefore, satellite fault detection is considered as one of the most important functions in GPS receivers. For the declining problem of the ability of fault detection for the traditional GPS satellite fault detection algorithm under the condition of small fault, a new GPS satellite fault detection algorithm based on wavelet analysis is proposed. The raw pseudorange measurements and the positioning data information are transformed by wavelet analysis and the data jumping point can be detected and identified through the different wavelet scales, and the satellite fault could be detected. Two kinds of GPS satellite fault detection methods are given in detail, and the advantages and disadvantages of them are compared. Validated by the real collected raw data from the GPS receiver, the results show that the wavelet analysis method can detect smaller mutations in a sequence of parameters, and the feasibility and effectiveness of applying the wavelet analysis algorithm in satellite fault detection for GPS receiver are verified.
\end{abstract}

Keywords: Global positioning system (GPS), Fault detection, Wavelet analysis, Pseudorange detection, Position detection

\section{Introduction}

The performance of global navigation satellite system (GNSS) includes positioning accuracy, availability, continuity and integrity. GNSS integrity is important for safety-critical applications, such as aircraft and missile applications. Integrity determines whether the navigation system is available. It is particularly important in some required high reliability positioning fields, especially air navigation. The integrity monitoring system is an inseparable part of a navigation system. Integrity can be able to detect and exclude faults that could cause risks to the accuracy and reliability, so that the GNSS navigation system can operate continuously without any degradation in performance. The main purpose of the satellite navigation receiver autonomous integrity monitoring is to detect the reliability of navigation results. When the positioning error is too large, the navigation system can not be used to provide navigation services. And the system warning should be required to give out timely [1]. At present, the fault detection algorithms include pseudorange snapshot algorithm and filtering algorithm. However, these methods exist some problems such as undetected the tiny fault, detection delay, the high false alarm rate [2-3]. Because the wavelet analysis has a good local properties of the signal in the time domain and the frequency domain, and there is a strong feature extraction [4], wavelet analysis can be used for GPS dynamical filter, GPS observation data de-noising and fault detection [5-8], and wavelet transform can be directly used to detect the observation signal singularity and to realize the system fault detection [9-10]. 
To address this above problem, a new fault detection algorithm using wavelet analysis was proposed. In the paper, the GPS receiver detected parameter data including pseudorange measurement and positioning data as signal processing objects that are processed by wavelet analysis respectively. Simulation analysis based on the real GPS data were verified.The paper is organized as follows. First, the theory of wavelet analysis is briefly reviewed. Then, the principle and general scheme of the algorithm followed by wavelet analysis approach to fault detection is presented. The next section is the description of the system and measurement equation of GPS receiver. Finally, the GPS receiver fault detection method is presented with numerical simulation and experiment.

\section{Fault Detection Theory Based on Wavelet Analysis}

Wavelet transform will do the $\tau$ displacement with the basic wavelet function $\psi(t)$, and do the inner product with $x(t)$ at different scales $s$.

$$
W T_{x}(s, \tau)=\frac{1}{s} \int_{-\infty}^{+\infty} x(t) \psi^{*}\left(\frac{t-\tau}{s}\right) d t, s>0
$$

Using wavelet transform multi-resolution or multi-scale characteristics, and the signal can be observed gradually from coarse to fine. Proper selection of the basic wavelet transform can make the time and frequency domain have the ability to characterize the local signal characteristics. Because wavelet transform features is prominent, it can be applied in fault detection [11-12]. Fault detection system is to detect the abnormal step point of system output data sequence. Since the system input and the amplitude, phase, frequency of output signal model is related with the fault [13-14]. When a faultoccurs, a signal processing and the feature extraction method for fault detection can be applied. Wavelet transform for the signal removes singular points caused by the input, and the rest of the singular point is the point of system failure.

\section{Wavelet Analysis Algorithm for GPS Fault Detection}

The problem of fault detection (FD) consists of making the decision on the presence or absence of faults in the GPS monitored system. In this paper, a fault detection method is designed using wavelet analysis algorithm. When the GPS satellite occur a fault, the pseudorange measurement data and the positioning data including longitude, latitude and altitude data will produce mutations, which can be detected by one or more mutual authentication using wavelet analysis processing pseudorange measurement and positioning data sequences. The extracted pseudorange measurement and positioning data sequence will produce the discontinuity information when GPS satellite occur a fault.

The GPS receiver state equation can be expressed using the following linear function.

$$
X_{k}=F_{k-1} X_{k-1}+w_{k-1}
$$

Where, $X_{k}=\left[r_{x}, r_{y}, r_{z}, \Delta \delta\right]^{T}$, and $\Delta \delta$ is the error of receiver clock with respect to satellite clock, and $F$ is the transfer matrix, which is the characteristic matrix in the stationary state, $w$ is the process noise.

The GPS measurement equation can be expressed using the following function.

$$
\rho^{i}(\mathrm{k})=\mathrm{R}^{i}(\mathrm{k})+\mathrm{c} \Delta \delta^{i}+T^{i}(\mathrm{k})+\mathrm{E}^{i}(\mathrm{k})+\varepsilon^{i}(\mathrm{k})
$$

Where, $\rho^{i}$ is the pseudorange between the coordinate $\left(\mathrm{r}_{x}, \mathrm{r}_{y}, \mathrm{r}_{z}\right)$ and the satellite $i$ with its coordinate $\left(\mathrm{s}_{x}, \mathrm{~s}_{y}, \mathrm{~s}_{z}\right), c$ stands for light speed, $\Delta \delta$ is the time offset, $T^{i}(\mathrm{k})$ is the troposphere error, $E^{i}$ is the ephemeris error, $\varepsilon$ is the code measurement noise, and $R^{i}$ stands for the distance between the satellite $i$ and the GPS receiver. 


$$
R^{i}=\sqrt{\left(\underset{\mathrm{r}-\mathrm{s}_{x}^{i}}{2}\right)+\left(\mathrm{r}^{2}{ }^{i}{ }_{y}^{2} \mathrm{~s}\right)+{ }_{x}\left(-\mathrm{r}^{i}\right.}
$$

The measurements are selected as follows. The coordinates of satellite $i\left(\mathrm{~s}_{x}, \mathrm{~s}_{y}, \mathrm{~s}_{z}\right)$, the pseudorange $\rho^{i}$ and the offset $\Delta \delta$ at each instant time, which can be attained from the measured data from the GPS receiver [15].

When the GPS satellite occur a failure, the pseudorange measurement data and the positioning data including longitude, latitude and altitude data will produce mutations, which can be detected by using wavelet analysis. The algorithm is described in detail as follows:

(1) Determine whether the system occur a fault at time t, the discrete wavelet transform is executed for the values sequence of the GPS measurement pseudorange and position data, and $W T_{p^{\prime}}(s, k)$ can be gotten.

(2) While using the pseudorange and position data parameters at time t-1, the sequence of $W T_{p^{\prime}}(t-1, t)(s, k)$ is gotten.

(3) $W T_{p^{\prime}}(t-1, t)(s, k)$ contains the vector gradient information. The $W T_{p^{\prime}}(s, k)$ and $W T_{p^{\prime}}(t-1, t)(s, k)$ are different. Then, the results eliminate gradient information and extract point information from a sequence of pseudo mutation.

(4) Using different wavelet functions for GPS receiver data on different hierarchy decomposition, detail coefficients and approximation coefficients are extracted. The reconstructing signal and the different layers of the upper layers of high-frequency signals, the original signal and the decomposition of different layers of signal components are given. Different wavelet functions and different decomposition hierarchy effects are adapted.

\section{Simulation Results of the GPS Fault Detection Algorithm}

The experimental data were obtained by the receiver N220 with the original observation data output. The initial value of the position is longitude $123^{\circ} 24.15436^{\prime}$ and latitude $41^{\circ} 55.53523$ ', and $418 \mathrm{~s}$ data is adaped from measurement data. There are six satellites for positioning, and the number of satellite is $3,15,18,19,21,26$. At the same time, after using another receiver module RCB-4H to monitor the output status of these satellites, the result shows that there is no failure, and the positioning result is reliable in a normal state. In order to simulate and verify whether the algorithm can correctly detect the fault satellite when a satellite fails, artificially add deviation time at 181s joined No. 19 satellite $200 \mathrm{~m}$ pseudorange bias, in addition, the obtained GPS 309s position data (longitude data and latitude data) artificially longitude data were added $0.003^{\circ}, 0.001^{\circ}$, $0.001^{\circ}$ deviation at time $30,71,159$, in latitude data were added to $0.0015^{\circ}, 0.003^{\circ}$, $0.001^{\circ}, 0.003^{\circ}$ bias at the time $17,49,190,222$.

\subsection{Experiment Results for Pseudorange Measurement Data Fault Detection}

Based on the above pseudorange measurement and position data, Daubechies and Symlets wavelet are used to detect a fault respectively, then a different decomposition hierarchy is selected to process GPS pseudorange measurement data and position data such as latitude and longitude, and the experimental results are as follows. 

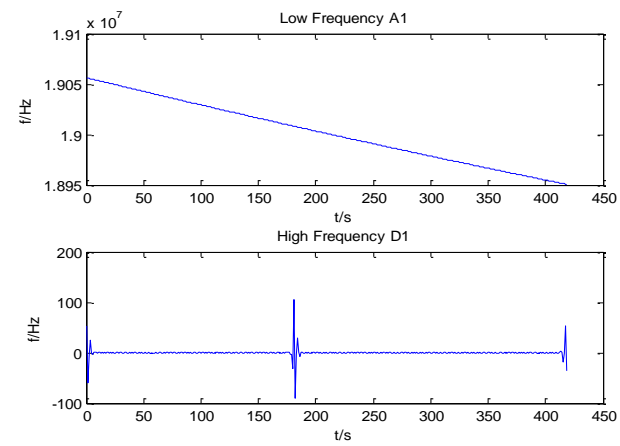

(a)

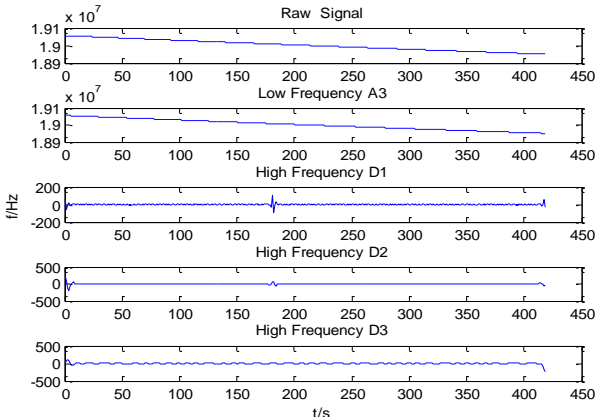

(b)

Figure 1. Pseudorange Measurement Data Fault Detection

Figure 1 (a) shows the low frequency component of the pseudorange data after one-dimensional discrete wavelet transform. The horizontal axis is the time step ( $t / \mathrm{s})$. Low-frequency component of the data contains the basic trend, and the high-frequency components in the pseudorange data show the detail information that can be more effective characteristics for the signal component.

Figure 1 (b) includes raw pseudorange data, low-frequency components, the first layer high-frequency component D1, the second layer high-frequency component D2 and the third layer high-frequency component D3. The horizontal axis is the time step (t/s). The first layer high-frequency component D1 and the second layer high frequency component D2 clearly show there are a clear point mutation in the time of 180s. Thus the method can effectively detect the fault data. By using symlets wavelet decomposition and reconstruction performance function, whose scales will increase with the increasing of layers. So during the system fault detection, it can take advantage of the wavelet analysis to detect the fault by different scale reconstruction, and the method can be also used to compare different scales for multiple fault detection.

\subsection{Experiment Results for Position data Fault Detection}
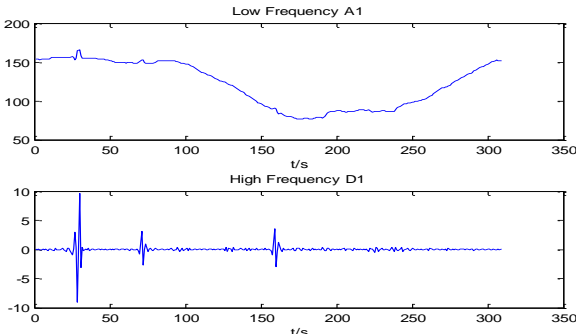

(a)
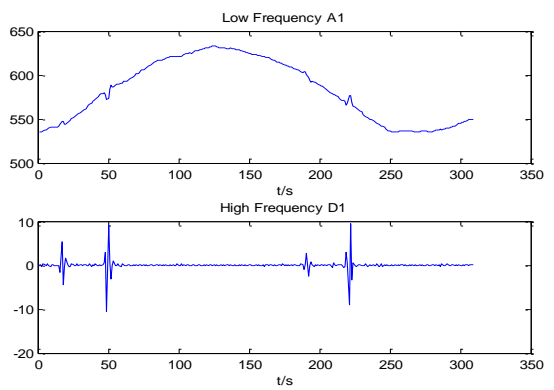

(c)

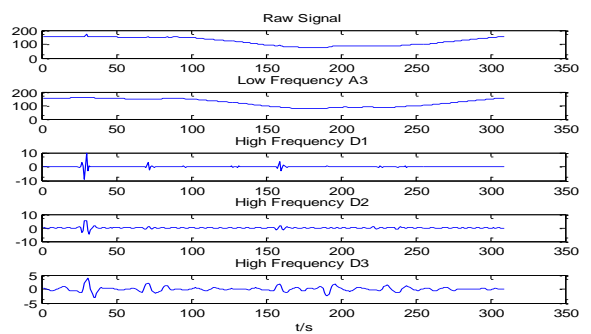

(b)

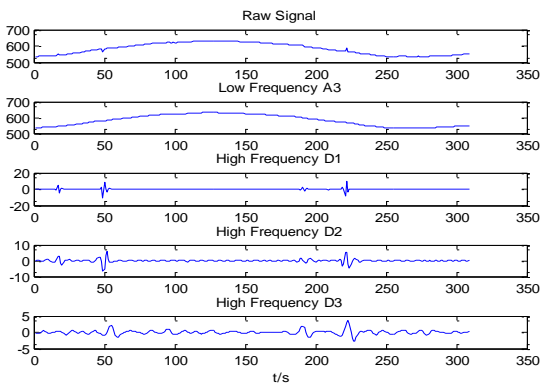

(d)

Figure 2. Position Data Fault Detection 
Figure 2 (a) is based on GPS position data in longitude data, after one-dimensional discrete wavelet analysis, the low frequency and high frequency components can be gotten. The horizontal axis is the time step (t/s), and the vertical axis is the part of longitude value. High-frequency components show the details of the specific information of data and through GPS longitude data point mutation, the fault can be detected.

Figure 2 (b) is the first layer high-frequency component D1 of longitude data obtained by the wavelet analysis, and the second layer high-frequency component D2 can be observed with the evidence of point mutations at three time of 30,71 and 159 .

Figure 2 (c) is based on GPS position data in latitude data, after one-dimensional discrete wavelet analysis, the low frequency and high frequency components can be gotten. The horizontal axis is the time step (t/s), and the vertical axis is the part of longitude value. High-frequency components show the details of the specific information and through GPS latitude data point mutation, the fault can be detected.

Figure 2 (d) is the first layer high-frequency component D1 of latitude data obtained by the wavelet analysis, the second layer high-frequency component D2 can be observed with the evidence of point mutations at four time of 17, 49, 190 and 222.

\subsection{Different Wavelet Decomposition Hierarchy Results and Analysis}

Although the wavelet analysis is widely used in signal processing fields, there are still two issues in the signal fault detection, namely the wavelet function selection and the best selection of wavelet decomposition hierarchy. Different wavelets have different time-frequency characteristics, and different wavelets will get different analysis results for the same signal. At the same time, different wavelet decomposition hierarchy will also affect the results of fault detection. In order to select the appropriate wavelet function and optimal decomposition hierarchy, the following simulations are done.

\subsubsection{Selection of the Wavelet Function}

In the experiments, the symlets wavelet and Daubechies wavelet decomposition are adapted to process GPS pseudorange data and position data, and the results are shown in Figure 3. The horizontal axis is the time step (t/s).
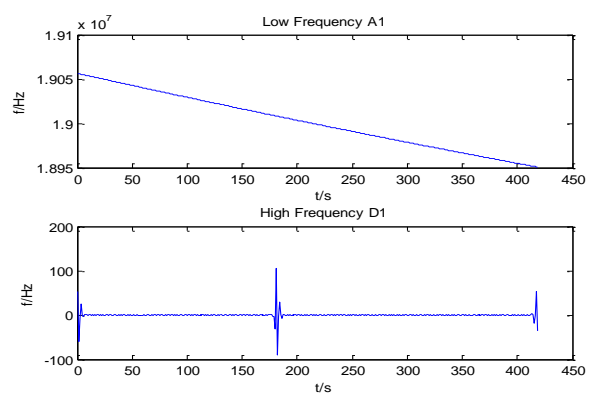

(a)
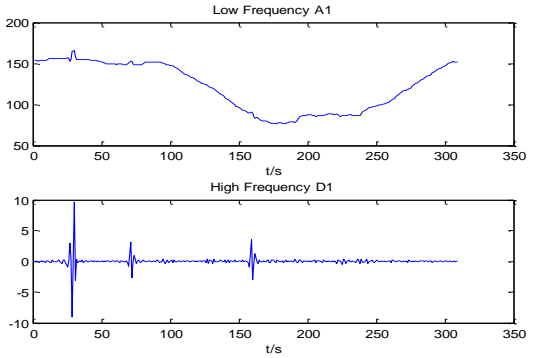

(c)
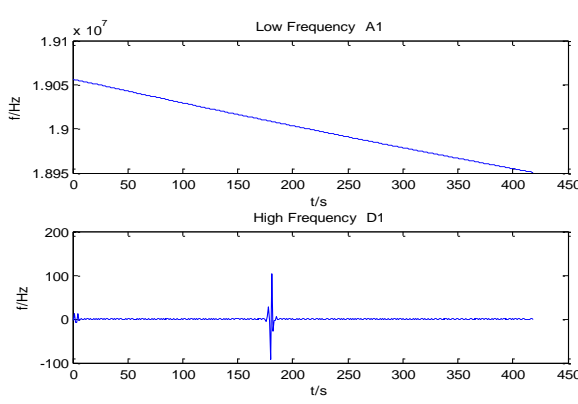

(b)
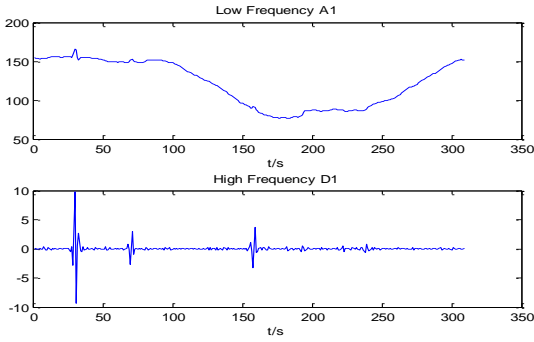

(d)

Figure 3. Comparison of Different Wavelet Decomposition Function 
The wavelet function sym5 is used in Figure 3 (a) for pseudorange data decomposition. The db4 wavelet function is used in Figure 3 (b) for pseudorange data decomposition. The wavelet function sym5 is used in Figure 3 (c) for longitude data decomposition. The db4 wavelet function is used in Figure 3 (d) for longitude data decomposition. The reconstruction error is shown in Table 1.

Table 1. Decomposition Reconstruction Error

\begin{tabular}{cll}
\hline Wavelet function & Pseudorange & Longitude \\
\hline Sym5 & $5.2149 \mathrm{e}-007$ & $4.9261 \mathrm{e}-007$ \\
\hline Db4 & $1.1293 \mathrm{e}-011$ & $1.0933 \mathrm{e}-010$ \\
\hline
\end{tabular}

Table 1 show that the error is smaller by using Daubechies wavelet for GPS pseudorange and longitude data, and Daubechies wavelet is more suitable for GPS satellite fault detection.

\subsubsection{Selection of the Wavelet Decomposition Hierarchy}

The wavelet function symlets are used to process pseudorange measurement data and position data. The results are shown in Figure 4. The horizontal axis is the time step $(\mathrm{t} / \mathrm{s})$, and the vertical axis is the pseudorange value, and the unit is meter.

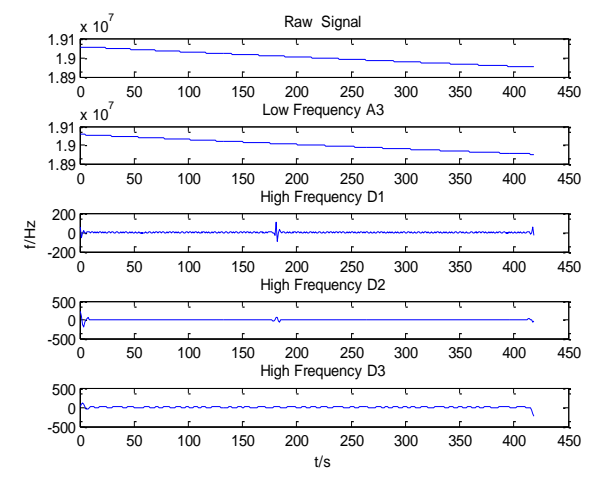

(a)

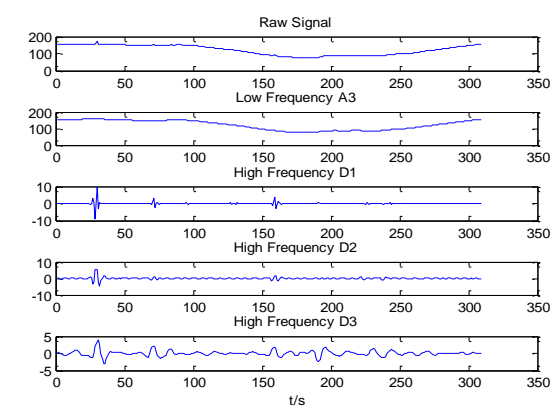

(c)

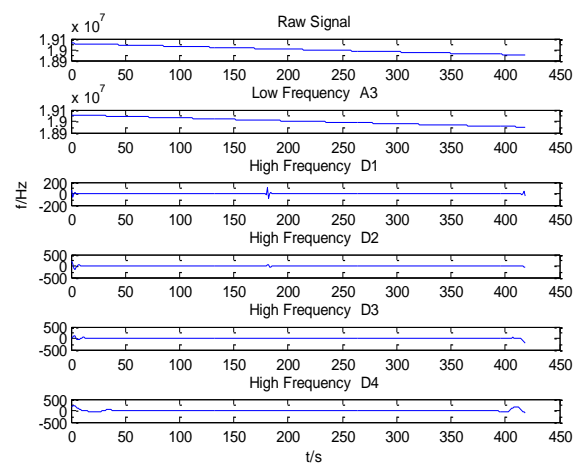

(b)

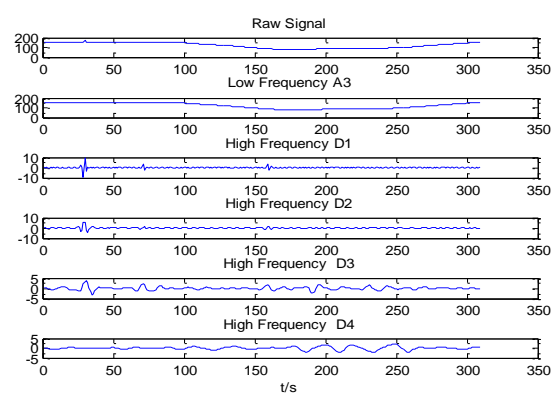

(d)

Figure 4. Comparison of Different Decomposition Hierarchy

The three hierarchies decomposing of sym5 wavelet function is used in Figure 4 (a) for pseudorange measurement data. And the four hierarchies decomposing of sym5 wavelet function is used in Figure 4 (b) for pseudorange measurement data. The three hierarchies decomposing of sym5 wavelet function is used in Figure 4 (c) for longitude data. The four hierarchies decomposing of sym5 wavelet function is used in Figure 4 (a) for longitude data. The reconstruction errors are shown in Table 2. 
Table 2. Reconstruction Error for Different Hierarchy

\begin{tabular}{lll}
\hline Hierarchy & Pseudorange & Longitude \\
\hline Three & $5.2149 \mathrm{e}-007$ & $1.1293 \mathrm{e}-011$ \\
\hline Four & $6.6930 \mathrm{e}-007$ & $1.3974 \mathrm{e}-011$ \\
\hline
\end{tabular}

Table 2 shows that the decomposition and reconstruction error is smaller when three decomposition hierarchies of the pseudorange GPS data and longitude data are adapted, and three hierarchies wavelet analysis is more conducive to detect the fault of GPS satellite.

\section{Conclusions}

Due to the advantages of wavelet analysis for fault detection, a new GPS fault detection method by using wavelet analysis was proposed for GPS navigation receiver. The fault of GPS satellite can be detected by using pseudorange measurement data and position data. The Symlets wavelet and Daubechies wavelet decomposition are adapted to process GPS pseudorange data and position data. Meanwhile, the selection of wavelet decomposition hierarchy and the selection function of wavelet fault detection are analyzed by the numerical simulation in detail. The different decomposition hierarchy and the reconstruction errors are compared respectively. Validated by the real collected raw data from the GPS receiver NS220, the results show that the wavelet analysis method can detect smaller mutations in a sequence of parameters and improve the accuracy of fault detection. Based on the simulation results, the feasibility and effectiveness of applying the wavelet analysis algorithm in the GPS satellite fault detection is verified.

\section{Acknowledgments}

This research was supported by the "Airborne GPS receiver autonomous integrity monitoring (RAIM) Based on Particle Filter Algorithm" through the National Natural Science Foundation of China (No.61101161), The Aeronautical Science Foundation of China (No. 2011ZC54010) and the Joint Funds of the Natural Science Foundation of Liaoning Province(2013024003).

\section{References}

[1] L. Yang, N. L. Knight, Y. Li and C. Rizos, "Optimal Fault Detection and Exclusion Applied in GNSS Positioning", Journal of Navigation, vol. 66, no. 5, (2013), pp. 683-700.

[2] N. L. Knight and J. Wang, "A Comparison of Outlier Detection Procedures and Robust Estimation Methods in GPS Positioning”, Journal of navigation, vol. 62, no. 4, (2009), pp. 699-709.

[3] J. Ahn, D. H. Won, M. Heo, Rosihan, Y. J. Lee, G. W. Nam and S. Sung, "GPS Integrity Monitoring Method Using Auxiliary Nonlinear Filters with Log Likelihood Ratio Test Approach”, Journal of Electrical Engineering \& Technology, vol. 6, no. 4, (2011), pp. 563 572.

[4] M. Joerger and B. Pervan, "Integrity risk of kalman filter-based RAIM", Proceedings of the 24th International Technical Meeting of The Satellite Division of the Institute of Navigation ION, (2011) September 19-23, Portland, USA.

[5] X. H. Xu, C. S. Yang and R. H. Liu, "Review and Prospect of GNSS Receiver Autonomous Integrity Monitoring”, Acta Aeronautica ET Astronautica Sinica, vol. 34, no. 3, (2013), pp. 451-463.

[6] A. G. Hafez, E. Ghamry, H. Yayama and K. Yumoto, "A wavelet spectral analysis technique for automatic detection of geomagnetic sudden commencements", IEEE Trans. On Geosciences and Remote sensing, vol. 50, no. 11, (2012), pp. 4503-4512.

[7] M. Yu, H. Guo and C. W. Zou, "Application of wavelet analysis to GPS deformation monitoring", IEEE PLANS, Position Location and Navigation Symposium, (2006) April 25-27, California, USA.

[8] J. Z. Wu, "De-noising of GPS observations with wavelet threshold method", Journal of Geodesy and Geodynamics, vol. 29, no. 4, (2009), pp. 79-82.

[9] H. L. Li, H. Fang, J. Tang and J. Zhang, "GPS Dynamic Filter Algorithm Based on Lifting Wavelet Transformation", Journal of Transportation Systems Engineering and Information Technology, vol. 13, no. 1, (2013), pp. 43-48. 
[10] S. H. Xu, J. H. Wu and Z. Q Hu, "Research on wavelet transform method for the fault-detection of the integrated navigation system”, Journal of Astronautics, vol. 24, no. 1, (2003), pp. 111-114.

[11] M. L. Cao and P. Y. Cui, "Information Fusion Technology of INS/GPS Navigation System Based on Wavelet Fault Detection", Journal of Astronautics, vol. 30, no. 5, (2009), pp. 1185-1890.

[12] Q. Xia, S. Y. Hao and C. Chang, "A Reserch on Failure Detection of Wavelet Transformation Based RAIM Algorithm”, Computer Measurement \& Control, vol. 22, no. 5, (2014), pp. 1320-1322.

[13] F. B. Cheng, B. P. Tang and Y. M. Zhong, "Optimal Morlet wavelet-based and SVD filtering denoising methods and fault diagnosis applications", Journal of Vibration and Shock, vol. 27, no. 2, (2008), pp. 91-94.

[14] L. L. Liu, H. Y. Wen and B. Liu, "Mitigation of systematic errors of GPS positioning based on wavelet denoise", 2nd WRI Global Congress on Intelligent Systems, (2010) December 16-17, Wuhan, China.

[15] E. Kaplan and C Hegarty, "Understanding GPS: Principles and Applications", Second Edition. USA: Artech House Inc., (2006).

\section{Authors}

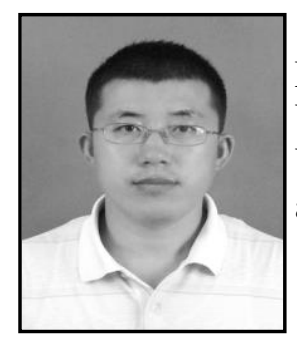

Ershen Wang, born in 1980, He received Ph. D. degree in Dalian Maritime University in 2009. He is pursuing post-doctoral in Beihang University. $\mathrm{He}$ is a associate professor in Shenyang Aerospace University. His main research interests are GNSS fault detection and avionics, etc.

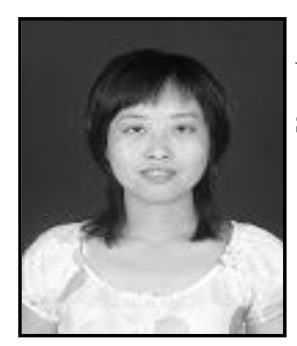

Tao Pang, born in 1976, she received Ph. D. degree in Beijing University of Technology. Her main research interest is intelligent signal processing.

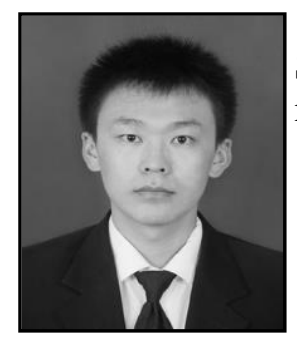

Yongming Yang, Born in 1987, he is a graduate student in Shenyang Aerospace University .His main research interest is the research of GPS principle. 